\title{
Intersubband Relaxation in CdSe Colloidal Quantum
}

\section{Wells}

Benjamin T. Diroll ${ }^{1 *}$ and Richard D. Schaller ${ }^{1,2}$

${ }^{1}$ Center for Nanoscale Materials, Argonne National Laboratory, Lemont, IL 60439

${ }^{2}$ Department of Chemistry, Northwestern University, Evanston, IL 60208

*bdiroll@anl.gov 


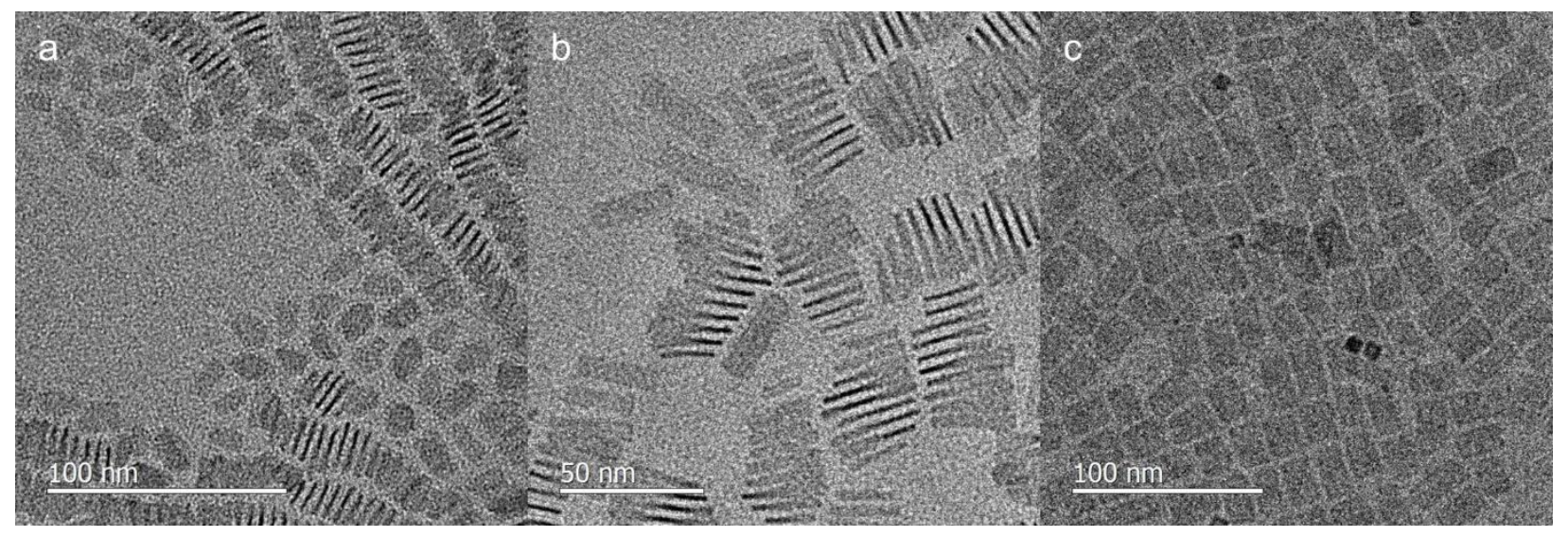

Figure S1. Typical TEM micrographs of (a) 4.5 ML, (b) 5.5 ML, and (c) 6.5 ML CdSe CQWs.

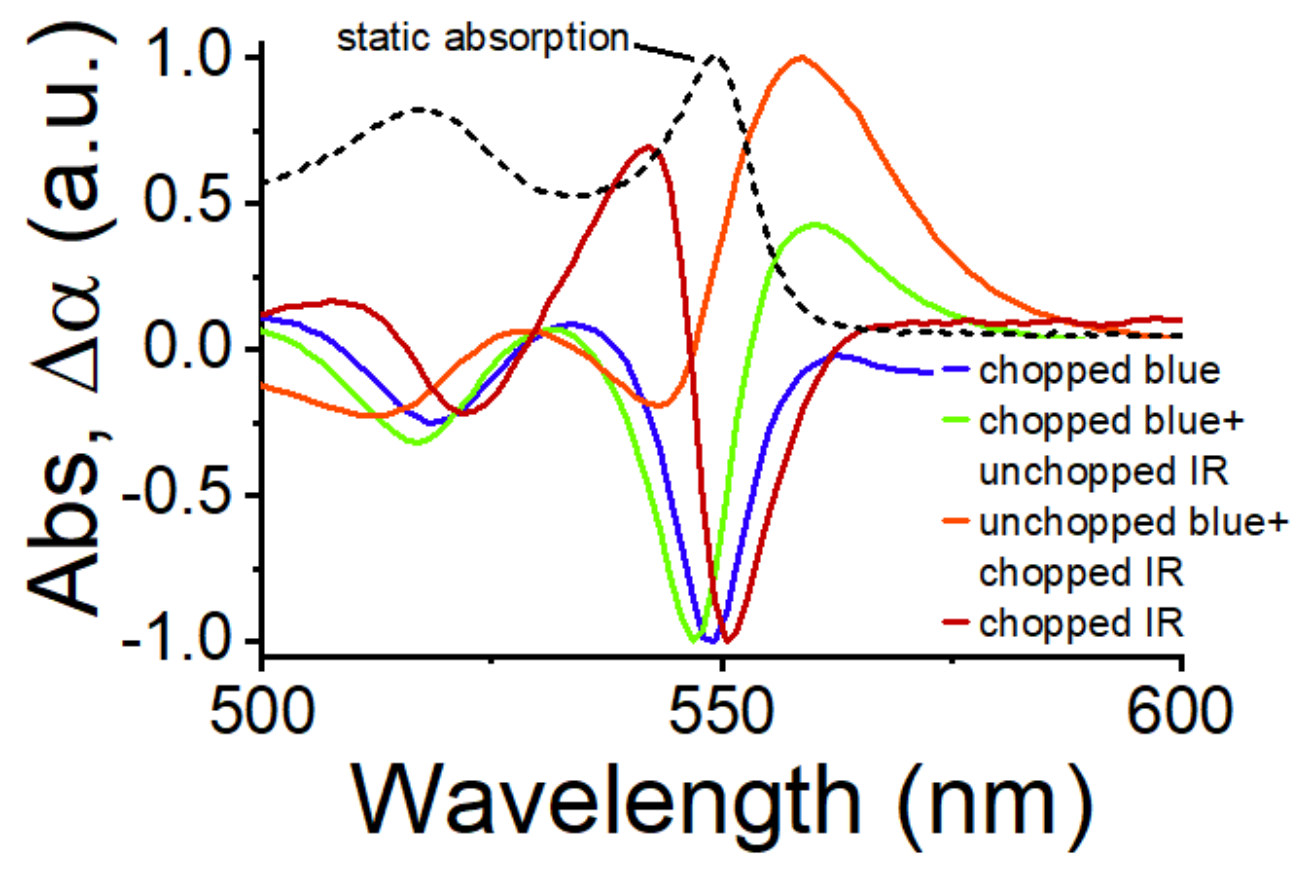

Figure S2. Normalized linecuts of two-dimensional transient absorption and pump-push-probe data with a dashed line showing the static absorption of the 5.5 ML CQW sample on which measurements were performed. Data are derived from Figure 1 in main text. 


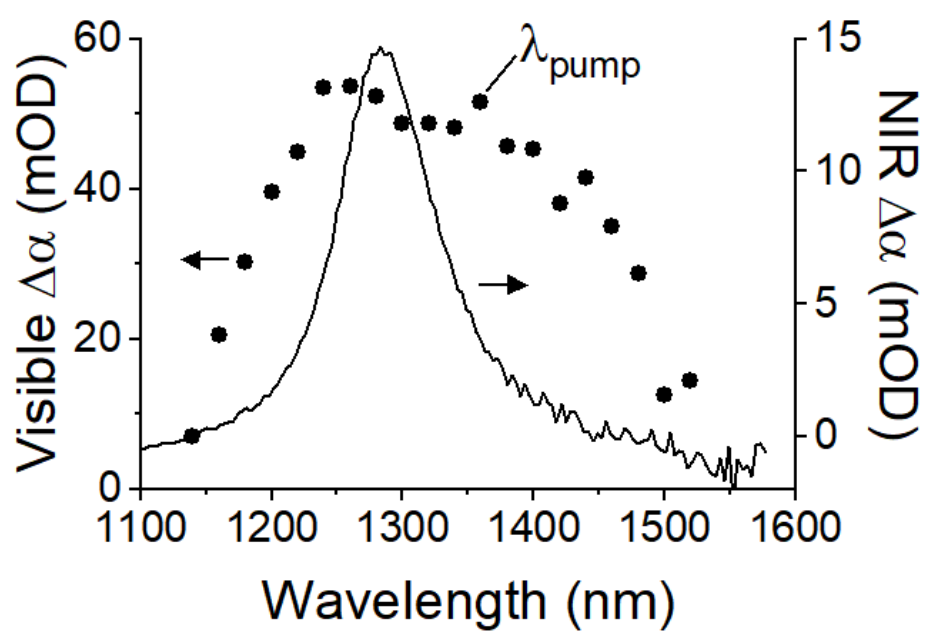

Figure S3. Action spectrum of pump-push-probe measurements (circles, left axis) overlaid on the intersubband absorption of a 5.5 ML CQW (solid line, right axis). The peak of visible $\Delta \alpha$ signal of pump-push-probe measurements for a fixed pump fluence $\left(100 \mu \mathrm{J} \cdot \mathrm{cm}^{-2}\right)$, pump-push delay $(50$ ps), and push fluence $\left(600 \mu \mathrm{J} \cdot \mathrm{cm}^{-2}\right)$, varying the push photon OPA wavelength setting (x-axis). True OPA output was not measured spectrally.

Table S1. Sizing statistics of CQW samples used in this work.

\begin{tabular}{lllll}
\hline Sample & Length $(\mathrm{nm})$ & Length deviation $(\mathrm{nm})$ & Width $(\mathrm{nm})$ & Width deviation $(\mathrm{nm})$ \\
\hline $4.5 \mathrm{ML}-1$ & 15.5 & 1.5 & 8.7 & 1.2 \\
$4.5 \mathrm{ML}-2$ & 17.9 & 2.1 & 9.5 & 1.0 \\
$4.5 \mathrm{ML}-3$ & 18.4 & 1.7 & 4.7 & 0.9 \\
$4.5 \mathrm{ML}-4$ & 16.4 & 2.6 & 7.9 & 1.2 \\
$4.5 \mathrm{ML}-5$ & 9.7 & 1.3 & 4.9 & 0.8 \\
$4.5 \mathrm{ML}-6$ & 27.5 & 3.5 & 9.5 & 1.3 \\
$5.5 \mathrm{ML}-1$ & 26.9 & 3.0 & 9.0 & 1.0 \\
$5.5 \mathrm{ML}-2$ & 28.9 & 4.2 & 10.0 & 1.5 \\
$5.5 \mathrm{ML}-3$ & 29.2 & 4.3 & 10.1 & 1.9 \\
$5.5 \mathrm{ML}-4$ & 30.6 & 3.5 & 5.7 & 0.9 \\
$5.5 \mathrm{ML}-5$ & 34.9 & 4.6 & 8.2 & 1.4 \\
$5.5 \mathrm{ML}-6$ & 25.6 & 3.4 & 8.9 & 1.5
\end{tabular}



6.5ML-1 $\quad 11.5$
1.2
9.0
1.0
6.5ML-2 24.6
2.2
14.2
2.0
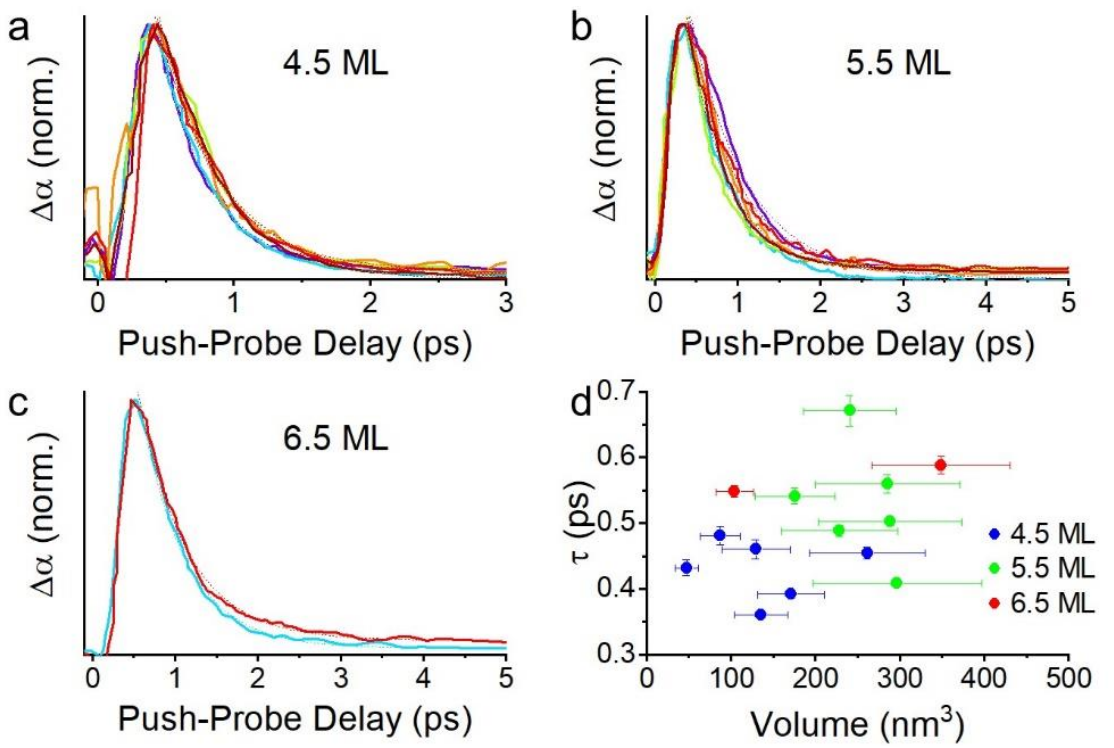

Figure S4. (a-c) Pump-push-probe dynamics of (a) 4.5 ML, (b) 5.5 ML, and (c) 6.5 ML CdSe CQW samples. Exponential fits are shown in dashed lines. (d) Fitted exponential relaxation times plotted versus CQW area for 4.5 ML (blue dots), 5.5 ML (green dots), and 6.5 ML (red dots). Horizontal errors are derived from TEM sizing analysis and vertical errors are from fitting error.
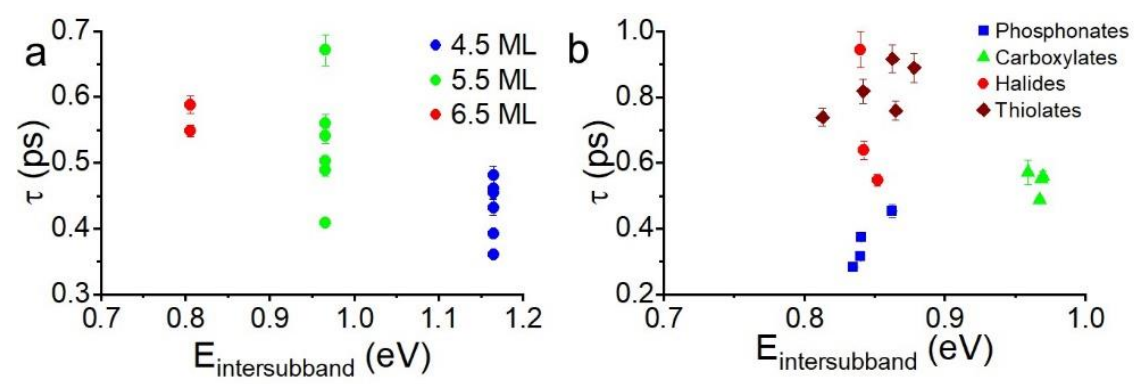

Figure S5. (a) Fitted intersubband relaxation times of several CQWs of varying sizes plotted against the intersubband energy. (b) Fitted intersubband relaxation times of $29.2 \mathrm{~nm} \times 10.1 \mathrm{~nm} 5.5$ ML CdSe CQW samples with different ligand exchanges. 

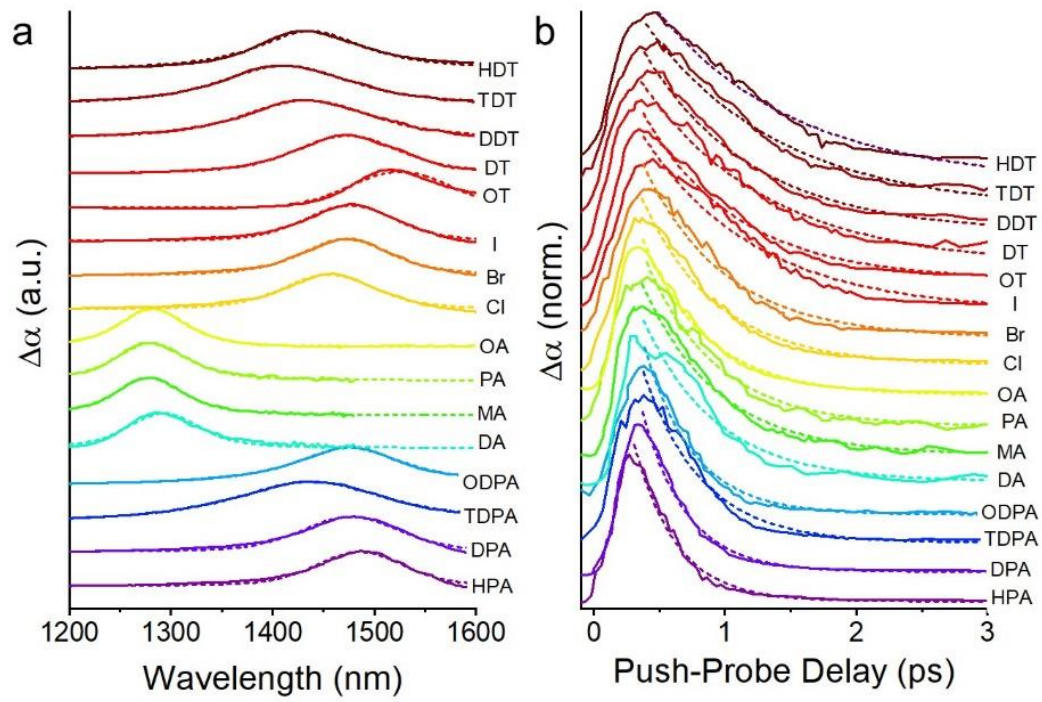

Figure S6. (a) Transient absorption spectra of 5.5 ML CdSe CQWs with different ligand coatings using a $400 \mathrm{~nm}$ pump taken at a $10 \mathrm{ps}$ pump-probe delay. (b) Pump-push-probe dynamics of 29.2 $\mathrm{nm} \times 10.1 \mathrm{~nm} 5.5 \mathrm{ML}$ CdSe CQWs with different ligand coatings. Samples were measured with $100 \mu \mathrm{J} \cdot \mathrm{cm}^{-2}$ pump fluence and $600 \mu \mathrm{J} \cdot \mathrm{cm}^{-2}$ push fluence with a 20 ps pump-push delay. Dashed lines show single exponential decay fits.
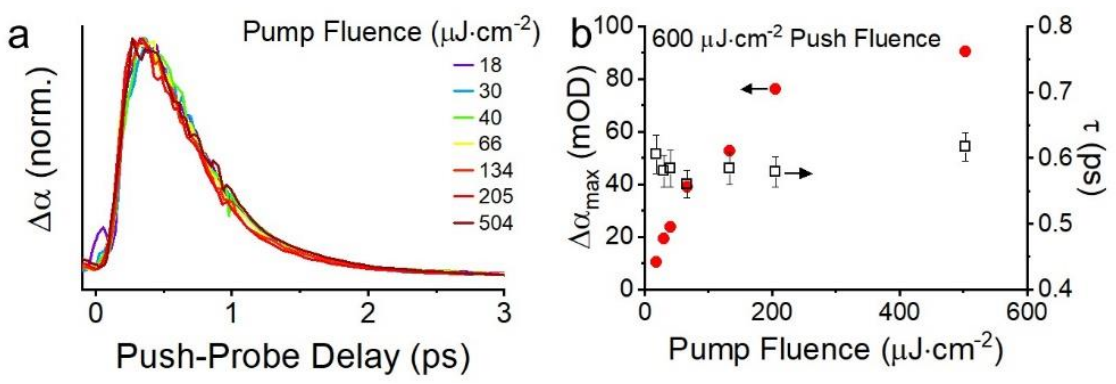

Figure S7. (a) Normalized pump-push-probe dynamics of $25.6 \mathrm{~nm} \times 8.9 \mathrm{~nm} 5.5 \mathrm{ML}$ CQWs examined as a function of pump fluence at a fixed push fluence $\left(600 \mu \mathrm{J} \cdot \mathrm{cm}^{-2}\right)$ and fixed pumppush delay (20 ps). (b) Peak signal at $558 \mathrm{~nm}$ (left) and relaxation time (right) of pump-push-probe measurements as a function of unchopped $400 \mathrm{~nm}$ pump fluence with a fixed 20 ps pump-push delay time and chopped $600 \mu \mathrm{J} \cdot \mathrm{cm}^{-2} 1270 \mathrm{~nm}$ push pulses. Error bars represent fitting error.
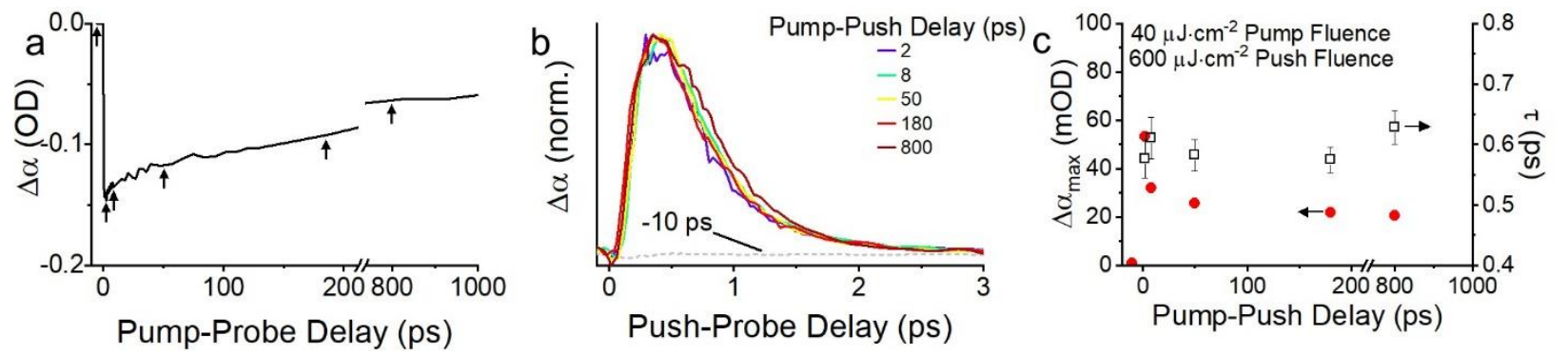

Figure S8. (a) Transient absorption of $25.6 \mathrm{~nm} \times 8.9 \mathrm{~nm} 5.5 \mathrm{ML}$ CdSe CQWs under $400 \mathrm{~nm}$ pump fluence of $40 \mu \mathrm{J} \cdot \mathrm{cm}^{-2}$. (b) Normalized pump-push-probe measurements on a $5.5 \mathrm{ML}$ CdSe CQW 
sample for several pump-push delay times, with $400 \mathrm{~nm}$ pump fluence of $40 \mu \mathrm{J} \cdot \mathrm{cm}^{-2}$ and $1280 \mathrm{~nm}$ push fluence of $600 \mu \mathrm{J} \cdot \mathrm{cm}^{-2}$. The data at -10 ps push-probe delay is not normalized. (c) Peak signal at $558 \mathrm{~nm}$ (left) and relaxation time (right) of pump-push-probe measurements as a function of pump-push delay time with a fixed unchopped $40 \mu \mathrm{J} \cdot \mathrm{cm}^{-2} 400 \mathrm{~nm}$ pump pulses and chopped 600 $\mu \mathrm{J} \cdot \mathrm{cm}^{-2} 1270 \mathrm{~nm}$ pump pulses. Error bars represent fitting error.
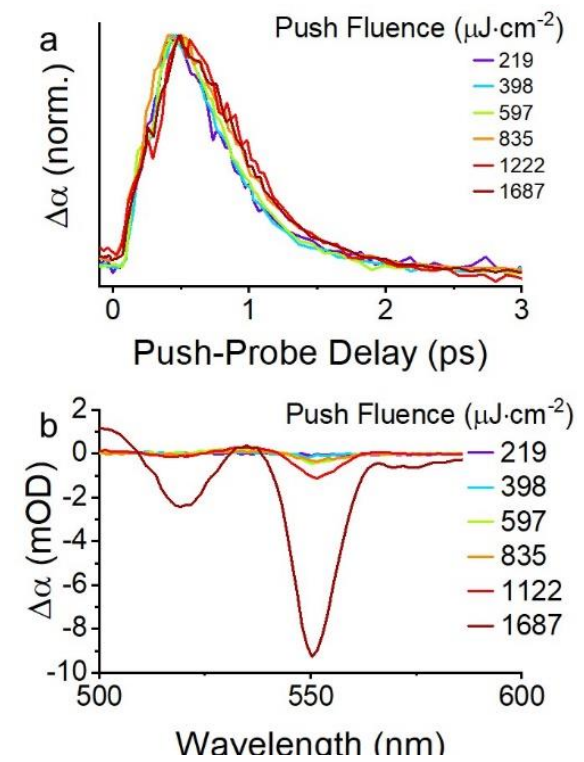

Figure S9. (a) Pump-push-probe dynamics of $25.6 \mathrm{~nm} \times 8.9 \mathrm{~nm} 5.5 \mathrm{ML}$ CQWs examined as a function of push fluence at a fixed pump fluence $\left(40 \mu \mathrm{J} \cdot \mathrm{cm}^{-2}\right)$ and fixed pump-push delay $(20 \mathrm{ps})$. Extracted lifetimes and peaks signals are found in Figure 3 of main text. (b) Pump-push-probe spectra of same at $10 \mathrm{ps}$ push-pump delay for several $1270 \mathrm{~nm}$ push fluences and fixed $40 \mu \mathrm{J} \cdot \mathrm{cm}^{-}$ ${ }^{2}$ pump fluence and 20 ps pump-push delay. Three-photon absorption is evident in the bleaching signal of the sample. 

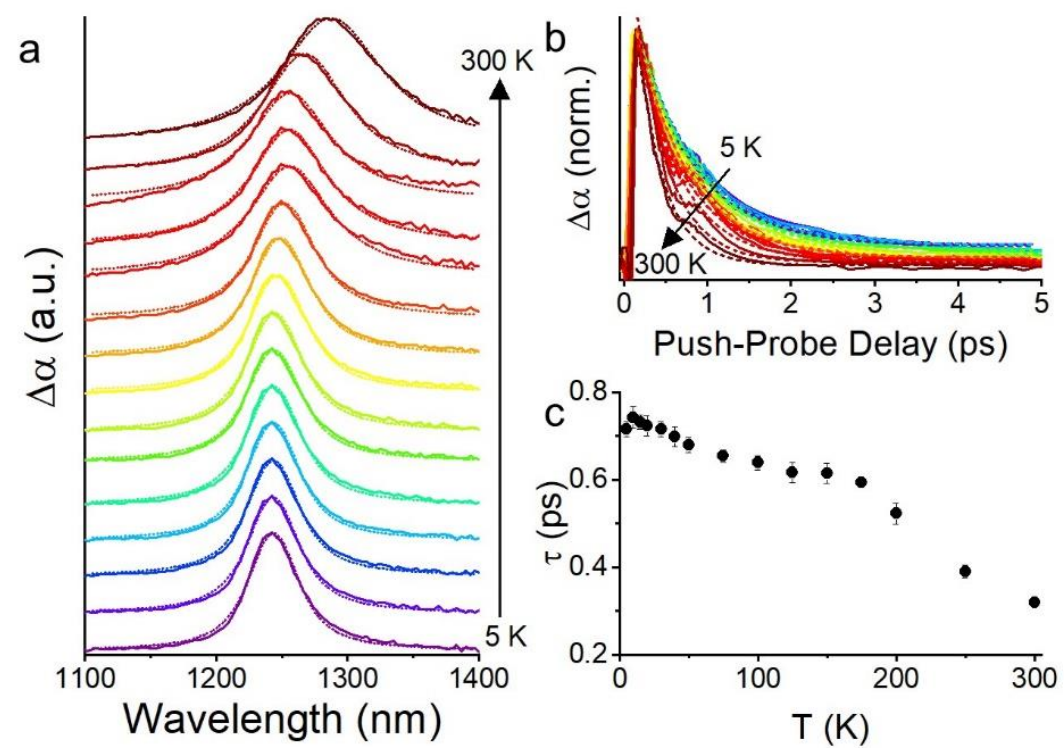

Figure S10. (a) Temperature-dependent intersubband absorption of a solid film of oleic-acid capped 5.5 ML CdSe CQWs observed at 10 ps pump-probe delay by transient absorption spectroscopy with $400 \mathrm{~nm}$ pump. Dashed lines show peak fits to estimate full-width at halfmaximum. (b) (b) Pump-push-probe dynamics of the same 5.5 ML CQWs for ascending temperatures from $5 \mathrm{~K}$ to $300 \mathrm{~K}$. Exponential decay fits are shown in dashed lines. (c) Fitted exponential relaxation times from temperature-dependent pump-push-probe measurements (black circles) plotted against temperature.

References

(1) She, C.; Fedin, I.; Dolzhnikov, D. S.; Dahlberg, P. D.; Engel, G. S.; Schaller, R. D.; Talapin, D. V. Red, Yellow, Green, and Blue Amplified Spontaneous Emission and Lasing Using Colloidal CdSe Nanoplatelets. ACS Nano 2015, 9, 9475-9485.

(2) Cho, W.; Kim, S.; Coropceanu, I.; Srivastava, V.; Diroll, B. T.; Hazarika, A.; Fedin, I.; Galli, G.; Schaller, R. D.; Talapin, D. V. Direct Synthesis of Six-Monolayer (1.9 nm) Thick Zinc-Blende CdSe Nanoplatelets Emitting at 585 nm. Chem. Mater. 2018, 30, 6957-6960.

(3) Antanovich, A.; Achtstein, A. W.; Matsukovich, A.; Prudnikau, A.; Bhaskar, P.; Gurin, V.; Molinari, M.; Artemyev, M. A Strain-Induced Exciton Transition Energy Shift in CdSe Nanoplatelets: The Impact of an Organic Ligand Shell. Nanoscale 2017, 9, 18042-18053.

(4) Dufour, M.; Qu, J.; Greboval, C.; Méthivier, C.; Lhuillier, E.; Ithurria, S. Halide Ligands to Release Strain in Cadmium Chalcogenide Nanoplatelets and Achieve High Brightness. ACS Nano 2019, 13, 5326-5334.

(5) Yeltik, A.; Delikanli, S.; Olutas, M.; Kelestemur, Y.; Guzelturk, B.; Demir, H. V. Experimental Determination of the Absorption Cross-Section and Molar Extinction Coefficient of Colloidal CdSe Nanoplatelets. J. Phys. Chem. C 2015, 119, 26768-26775.

(6) Hopper, T. R.; Gorodetsky, A.; Frost, J. M.; Müller, C.; Lovrincic, R.; Bakulin, A. A. Ultrafast Intraband Spectroscopy of Hot-Carrier Cooling in Lead-Halide Perovskites. ACS 
Energy Lett. 2018, 3, 2199-2205.

(7) Pandey, A.; Guyot-Sionnest, P. Slow Electron Cooling in Colloidal Quantum Dots. Science 2008, 322, 929-932.

(8) Guyot-Sionnest, P.; Wehrenberg, B.; Yu, D. Intraband Relaxation in CdSe Nanocrystals and the Strong Influence of the Surface Ligands. J. Chem. Phys. 2005, 123, 074709.

(9) Klimov, V. I. Quantization of Multiparticle Auger Rates in Semiconductor Quantum Dots. Science 2000, 287, 1011-1013.

(10) Blackburn, J. L.; Ellingson, R. J.; Mićić, O. I.; Nozik, A. J. Electron Relaxation in Colloidal InP Quantum Dots with Photogenerated Excitons or Chemically Injected Electrons. J. Phys. Chem. B 2003, 107, 102-109.

(11) Guyot-Sionnest, P.; Shim, M.; Matranga, C.; Hines, M. A. Intraband Relaxation in CdSe Quantum Dots. Phys. Rev. B 1999, 60, R2181-R2184. 